\title{
Coding algorithms for defining Charlson and Elixhauser co-morbidities in Read- coded databases
}

\author{
David Metcalfe ${ }^{1 *}$ D, James Masters ${ }^{1}$, Antonella Delmestri ${ }^{2}$, Andrew Judge ${ }^{2,3,4,5}$, Daniel Perry ${ }^{1}$, Cheryl Zogg ${ }^{1,6}$, \\ Belinda Gabbe ${ }^{7}$ and Matthew Costa ${ }^{1}$
}

\begin{abstract}
Background: Comorbidity measures, such as the Charlson Comorbidity Index (CCl) and Elixhauser Method (EM), are frequently used for risk-adjustment by healthcare researchers. This study sought to create CCI and EM lists of Read codes, which are standard terminology used in some large primary care databases. It also aimed to describe and compare the predictive properties of the $\mathrm{CCl}$ and EM amongst patients with hip fracture (and matched controls) in a large primary care administrative dataset.

Methods: Two researchers independently screened 111,929 individual Read codes to populate the $17 \mathrm{CCl}$ and 31 EM comorbidity categories. Patients with hip fractures were identified (together with age- and sex-matched controls) from UK primary care practices participating in the Clinical Practice Research Datalink (CPRD). The predictive properties of both comorbidity measures were explored in hip fracture and control populations using logistic regression models fitted with 30- and 365-day mortality as the dependent variables together with tests of equality for Receiver Operating Characteristic (ROC) curves.

Results: There were $5832 \mathrm{CCl}$ and 7156 EM comorbidity codes. The EM improved the ability of a logistic regression model (using age and sex as covariables) to predict 30-day mortality (AUROC 0.744 versus 0.686 ). The EM alone also outperformed the $\mathrm{CCl}(0.696$ versus 0.601$)$. Capturing comorbidities over a prolonged period only modestly improved the predictive value of either index: EM 1-year look-back 0.645 versus 5 -year 0.676 versus complete record 0.695 and $\mathrm{CCl} 0.574$ versus 0.591 versus 0.605 .

Conclusions: The comorbidity code lists may be used by future researchers to calculate $\mathrm{CCl}$ and EM using records from Read coded databases. The EM is preferable to the CCI but only marginal gains should be expected from incorporating comorbidities over a period longer than 1 year.
\end{abstract}

Keywords: Comorbidity, Read codes, Charlson, Elixhauser

\section{Background}

The comparison of patient outcomes between healthcare providers requires effective risk adjustment for patient characteristics. In particular, comorbidities are important predictors of outcome ${ }^{12}$. Comorbidity summary measures have been developed to help classify patients according to their overall disease burden [1-4].

\footnotetext{
* Correspondence: david.metcalfe@balliol.ox.ac.uk

'Oxford Trauma, Kadoorie Centre for Critical Care Research and Education, Nuffield Department of Orthopaedics, Rheumatology and Musculoskeletal Sciences (NDORMS), John Radcliffe Hospital, Headley Way, Oxford OX3 9BU, UK

Full list of author information is available at the end of the article
}

The most commonly used summary measure is the Charlson Comorbidity Index (CCI) [4]. Charlson et al. identified 17 diseases that optimally predict one-year mortality when assigned a weight between 1 (e.g. peripheral vascular disease) and 6 (e.g. metastatic cancer) [1]. Although the CCI is commonly used [4] and has been widely validated [5], it was developed in the 1980s and has been criticized as outdated [6]. A number of meta-analyses have found that an alternative summary measure proposed by Elixhauser et al. [2] has superior predictive properties ${ }^{3}{ }^{4}$. In particular, the Elixhauser Method (EM) predicts mortality more effectively than CCI amongst patients with

(c) The Author(s). 2019 Open Access This article is distributed under the terms of the Creative Commons Attribution 4.0 International License (http://creativecommons.org/licenses/by/4.0/), which permits unrestricted use, distribution, and reproduction in any medium, provided you give appropriate credit to the original author(s) and the source, provide a link to the Creative Commons license, and indicate if changes were made. The Creative Commons Public Domain Dedication waiver (http://creativecommons.org/publicdomain/zero/1.0/) applies to the data made available in this article, unless otherwise stated. 
fractures of the cervical spine [7] and proximal humerus [8]. However, although older adults with hip fractures have a high comorbid disease burden, it is unclear which summary measure optimally predicts mortality in this population. The EM is similar to the CCI (nine categories overlap the two measures: diabetes [uncomplicated and complicated], congestive heart failure, HIV, metastatic cancer, renal disease, chronic pulmonary disease, rheumatic disease, and peripheral vascular disease) but includes almost twice as many diagnostic categories [9].

A number of algorithms have been developed to determine CCI and EM from administrative databases based on ICD-9 [10-12] and ICD-10 [9] diagnostic codes. Although Khan et al [13] have developed an algorithm for calculating CCI in Read-coded databases; there is no equivalent translation for EM. This is important because Read codes are used by General Practitioners throughout the United Kingdom National Health Service (NHS) [14] and are the basis on which a number of national primary care datasets have developed. These include the Clinical Practice Research Datalink (CPRD) GOLD [15] and The Health Improvement Network (THIN) [16] databases.

The aims of this study were to: (1) develop coding algorithms for calculating CCI and EM in Read-coded databases, (2) describe the comorbidity characteristics of a hip fracture cohort with matched controls, and (3) compare the predictive properties of the CCI (both original and modified versions) and the EM.

\section{Methods \\ Defining co-morbidity algorithms}

The multi-step process for selecting comorbidity diagnostic codes is shown by Figs. 1 and 2. First, the 31 comorbidities defined by Elixhauser et al. [2] and 17 by Charlson et al. [1] were extracted from their original publications. The Charlson paper was supplemented with work by Deyo et al. [11] who previously translated the Charlson co-morbidities into ICD-9-CM codes. Each comorbidity category was presented together with its ICD-9-CM codes and a text interpretation of each code (exploded to show the full hierarchy of sub-codes) from the 6th edition of the International Classification of Diseases, 9th Revision, Clinical Modification (ICD-9-CM) [17]. This step was necessary because many Read terms are unstructured but the ICD-9-CM hierarchy acted as an aide memoire for diagnoses that might otherwise be missed. For example, clinicians trying to populate the Charlson category "Any malignancy, including leukaemia and lymphoma" might search for "lymphoma" but could inadvertently omit "mycosis fungoides" (represented by 7 separate Read codes) or "Letterer-Siwe disease" (5 Read codes). However, the researchers would encounter

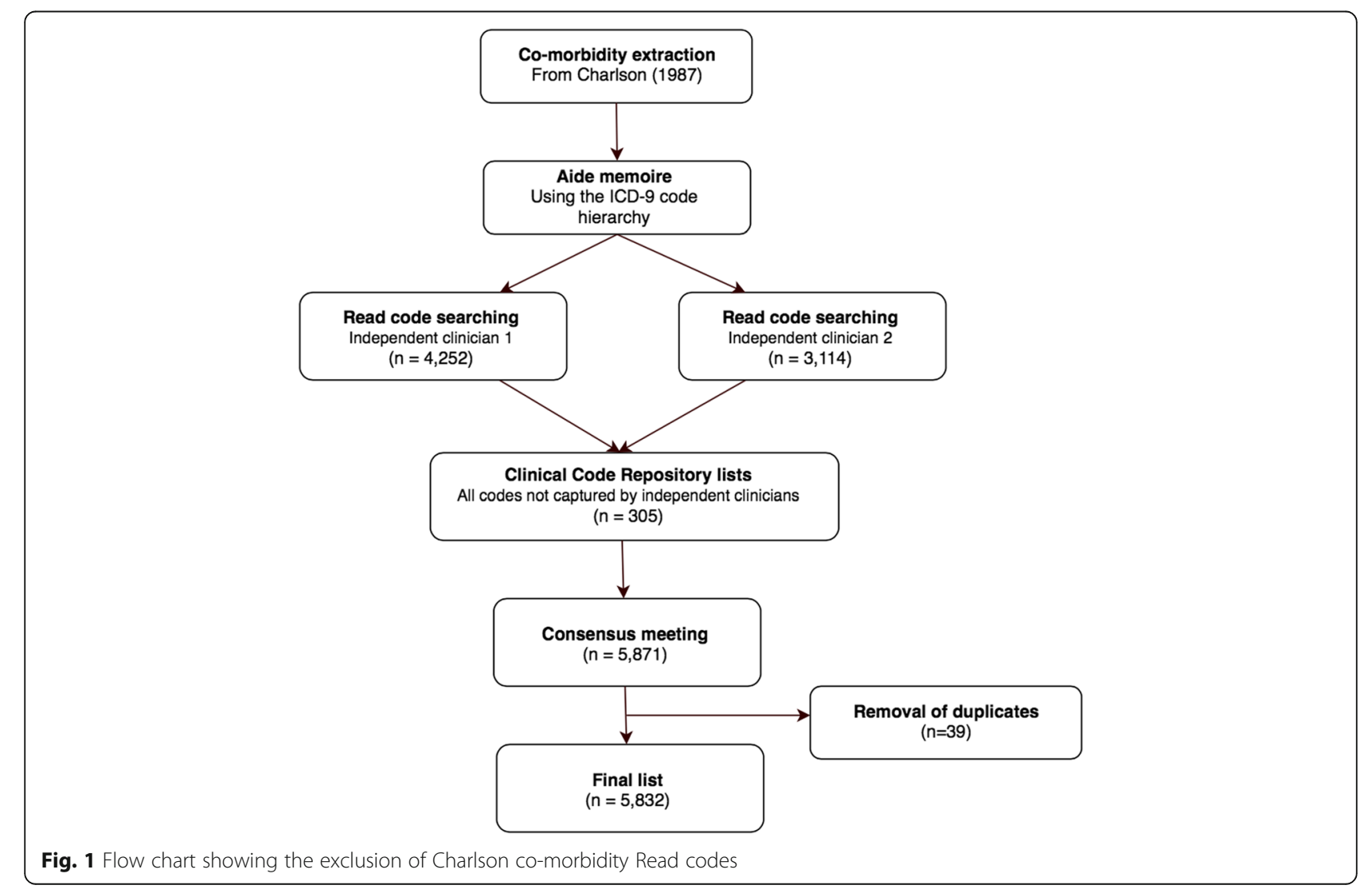




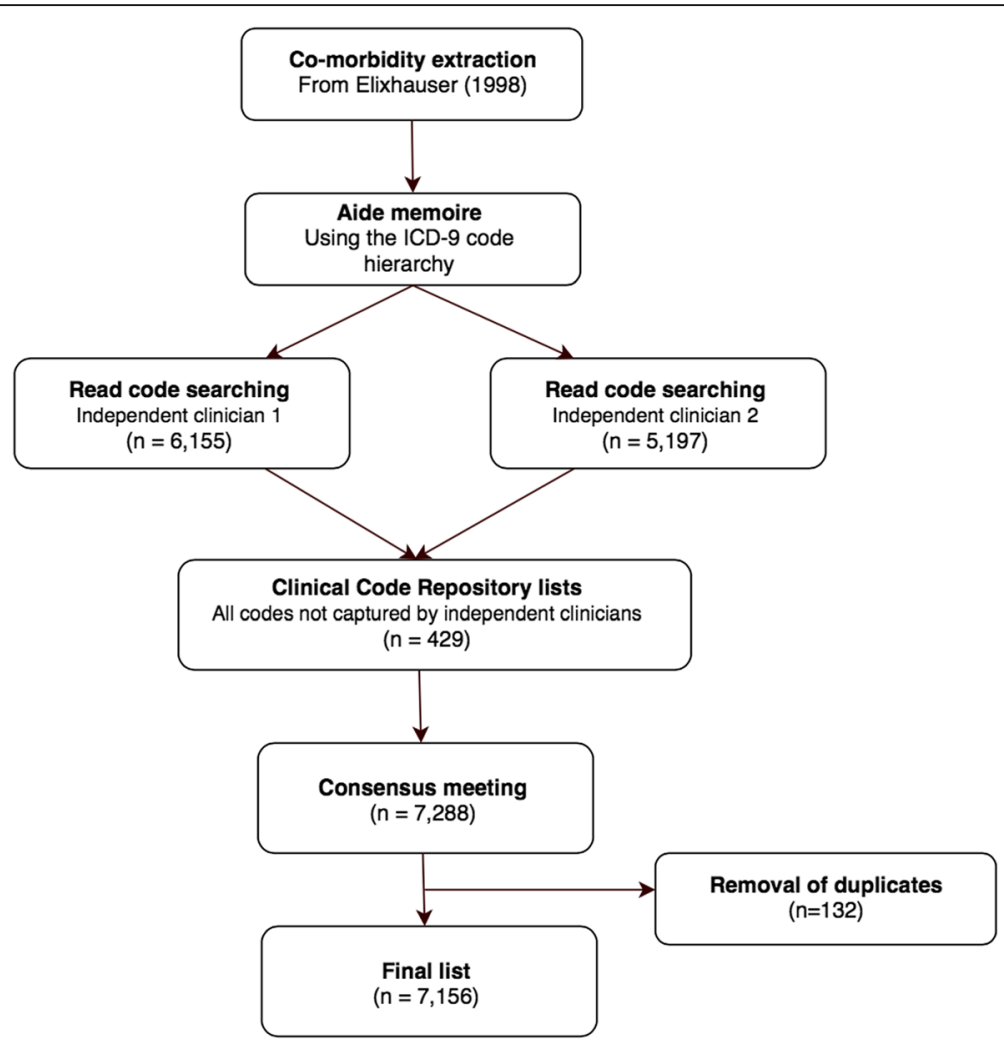

Fig. 2 Flow diagram showing the exclusion of Elixhauser co-morbidity Read codes

all three codes while working through the "Malignant neoplasm of lymphatic and hematopoietic tissue" chapter of ICD-9-CM. Similarly, even a specialist might search for "myeloid sarcoma" but not think to search for additional Read codes under "chloroma". They would however find "chloroma" listed under "myeloid sarcoma" in the ICD-9-CM hierarchy.

Two clinicians independently used the exploded ICD-9CM text codes to search all 111,929 Read terms within the CPRD Medical Dictionary [18]. The general search principles and assumptions agreed by the two code screeners are available in Additional file 3 The result of this process was that each screener developed a list of Read terms that corresponded to the ICD-9-CM codes recommended by Elixhauser [2] and Charlson/Deyo [1, 11].

In addition, the online ClinicalCodes Repository [19] was manually searched for all pre-existing Read code lists that pertained to each comorbidity category. Lists from 12 studies [20-30] were included from the ClinicalCodes Repository in addition to the CCI list previously developed by Khan et al [13]. The outcome of this process was that between two and six independent Read code lists were generated for each comorbidity category. The two clinicians then resolved discrepancies through discussion and with advice from sub-specialists where appropriate. A single list was generated for each comorbidity measure and duplicate entries deleted. A final logic check was performed by a single clinician.

\section{Co-morbidity characteristics of a hip fracture cohort}

The CPRD is an ongoing primary care database of medical records provided by General Practitioners [15]. It is owned by the Medicines and Healthcare products Regulatory Agency (MHRA) and collects data about more than 11.3 million patients from 674 individual GP practices. Approximately $6.9 \%$ of UK residents are currently represented by data in the CPRD and these are broadly representative of the wider UK population. General Practitioners in the UK maintain each patient's entire healthcare record and should receive correspondence (including Emergency Department correspondence, outpatient clinic letters, and hospital discharge summaries) from secondary care providers. Important events (e.g. hip fracture) and diagnoses (e.g. interstitial lung disease) should therefore be coded into the GP record even if the patient was treated in hospital.

A cohort of patients (cases) were identified from CPRD GOLD practices based on a first ever record of "hip fracture" occurring between 1st January 1999 and 9th October 2013. The diagnostic and procedural codes used to define this hip fracture cohort are presented in Additional file 4 In addition, the patients required at 
least 3 years up-to-standard (UTS) registration in CPRD GOLD prior to their hip fracture [15]. Age- and sexmatched controls were identified by the CPRD in a 2:1 ratio from patients registered with practices from 1st January 1996 onwards and with at least three preceding years UTS registration.

The specific variables extracted from the CPRD were age, sex, date of hip fracture diagnosis, and individual comorbidities. For the principal analysis, diagnostic codes were extracted from each patient's entire lifetime primary care record. We also planned sensitivity analyses that confined comorbidities to those recorded within 1and 5-years of the index hip fracture.

\section{Validating the predictive properties of the Elixhauser method}

The EM was tested against the CCI, using both the 17-item original version by Charlson et al [1] and the shorter 12item modification proposed by Quan et al [6]. We planned to report 30- and 365-day mortality. Kaplan-Meier plots were created for death within 365 days by categories of CCI and EM. Logistic regression models were fitted with 30and 365-day mortality as the dependent variables. The covariables were age (as a continuous variable) and sex, which is consistent with the approach taken by other studies designed to evaluate comorbidity summary measures. The subsequent analyses fitted multivariable logistic regression models with age and sex as well as either CCI or Elixhauser comorbidities as covariables. Stepwise variable selection techniques were not used. The comorbidity summary measures were then layered on top of this base model. Tests of equality for Receiver Operating Characteristic (ROC) areas were undertaken using the roccomp [31] module in Stata v.15.0 (College Station, TX, USA). Although summary tables were produced to show the number of EM comorbidities in each group, these were included as separate independent variables within regression models in the manner proposed by Elixhauser et al. [2]. The principal analysis used CCI calculated using the weights originally proposed by Charlson et al. [1]. We reported the predictive properties of the EM and CCI in both diseased (i.e. hip fracture) and non-diseased (age- and sex-matched control) populations. Importantly, we undertook analyses of cases and controls separately and did not plan to utilize a case-control design. In addition, we undertook sensitivity analyses limited to comorbidities documented 1- and 5-years before the index hip fracture as some researchers may find themselves working with cuts of data that are limited in time. The principal analysis used all comorbidies documented at any time in each patient's complete medical record.

\section{Information governance}

Ethical approval was not sought in line with the latest Governance Arrangements for Research Ethics
Committees (GafREC) guidance [32]. Approval to use the data was provided by the Independent Scientific Advisory Committee (ISAC) at the MHRA (ISAC Protocol No. 13_069RA). Personal data was processed under Articles $6(1)(f)$ and $9(1)(f)$ of the General Data Protection Regulation (EU 2016/6709).

\section{Results \\ Defining an Elixhauser coding algorithm}

Figures 1 and 2 show the number of diagnoses identified and eliminated for CCI and EM respectively. The final lists included 5832 individual codes representing CCI comorbidities (Additional file 1) and 7156 EM comorbidities (Additional file 2).

\section{Comorbidity characteristics of a patient cohort}

The linked dataset included 13,974 patients with hip fractures and 26,860 age- and sex-matched controls. The median age across the cohort was 82 years (interquartile range [IQR]: $75-87$ years and $75.1 \%$ were female. The distribution of comorbidities within the cohort according to Charlson and Elixhauser are shown in Figs. 3 and 4. Table 1 shows that $27.6 \%$ of hip fracture patients did not have any CCI co-morbidities recorded; only $9.2 \%$ did not have EM conditions recorded. The median CCI was 1 (interquartile range [IQR] 0-3) and EM 2 (IQR 1-4).

30-day mortality The EM improved the ability of a logistic regression model (using age and sex as covariables) to predict 30-day mortality (AUROC 0.744 [95\% CI 0.727 to 0.760 ] versus 0.686 [0.668 to 0.705$]$ ) among cases. The EM alone performed better than CCI in predicting 30-day mortality (AUROC 0.696 [95\% CI 0.677 to 0.714 ] versus 0.601 [0.582 to 0.619]). Similar findings were observed within the control population: EM + base model AUROC 0.771 (95\% CI 0.743 to 0.800 ) versus EM alone 0.709 (0.678 to 0.740 ) and EM 0.726 (0.692 to 0.760 ) versus CCI 0.649 ( 0.614 to 0.683 ).

365-day mortality Fig. 5 shows that the EM modestly improved the ability of the base regression model to predict 365-day mortality (AUROC 0.726 [95\% CI 0.716 to 0.735 ] versus 0.676 [0.665 to 0.687$]$ ) amongst cases. Fig. 6 shows that the CCI only performed marginally better than the base model: AUROC 0.676 [95\% CI 0.665 to 0.687]. The EM alone performed better than the CCI (0.672 [95\% CI 0.661 to 0.683$]$ ) versus 0.611 [95\% CI 0.600 to 0.622$])$. Similar findings were observed within the control population: EM + base model AUROC 0.750 (95\% CI 0.740 to 0.759 ) versus EM alone 0.700 (0.690 to 0.710 ) and EM 0.696 (0.685 to 0.707 ) versus CCI 0.635 (0.622 to 0.645$)$.

\section{Use of diagnoses recorded over different durations}

The proportion of patients with a CCI of zero decreased, consistent with expectations, as the length of time over 


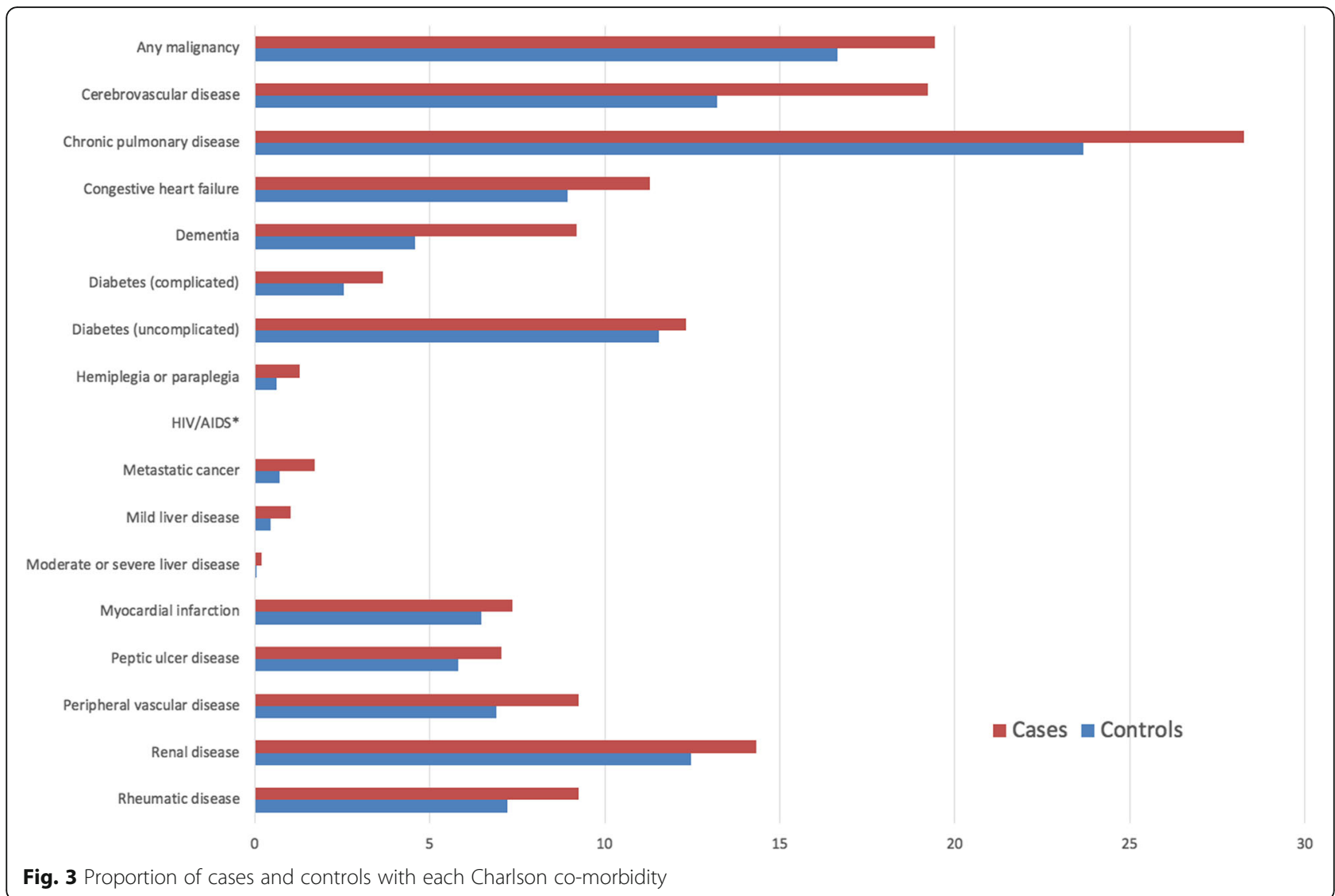

which diagnostic codes were assessed increased, e.g. $64.1 \%$ cases had $\mathrm{CCI}=0$ at 1 -year versus $42.9 \%$ at 5 -year and $27.6 \%$ using the complete record. A similar trend was observed for the number of EM co-morbidities, although the difference between 1 and 5 years was less marked (cases 1 -year $48.4 \%$ versus 5 -year $48.3 \%$ and complete record 9.2\%). The increased capture of diagnostic codes by using the complete primary care record only modestly improved the predictive value of either the CCI (1-year AUROC 0.574 [95\% CI 0.555 to 0.572] versus 5-year 0.591 [ 0.572 to 0.610 ] versus complete record 0.605 [0.586 to 0.623$]$ ) or the EM (1-year 0.645 [0.625 to 0.664 ] versus 5 -year 0.676 [0.657 to 0.696 ] versus complete record 0.695 [0.677 to 0.714 ) for 30-day mortality amongst cases. Similar results were observed amongst controls (Additional file 5).

\section{Use of updated Charlson comorbidity index}

The updated CCI described by Quan et al [6] performed similarly in this population to the original index, both for 30- (AUROC 0.716 [95\% CI 0.699 to 0.733] versus 0.704 [0.686 to 0.721$]$ ), and 365-day mortality (AUROC 0.713 [95\% CI 0.703 to 7.23 ] versus 0.700 [0.690 to $0.710])$. The modified weights proposed by Quan et al
[6] have been included as an additional column in Additional file 1.

Use of Charlson co-morbidities as individual co-variables The Charlson co-morbidities performed marginally better for predicting 30-day mortality when included as independent co-variables than when used as a single index, both amongst cases (individual co-variables 0.632 [0.612-0.652] versus CCI 0.612 [0.594-0.631]) and controls (0.679 [0.642-0.715] versus 0.660 [0.625-0.693]). This also held for predicting 365-day mortality amongst cases (CM 0.638 [0.627-0.649] versus CCI 0.616 [0.605$0.628]$ ) and controls (individual co-variables 0.662 [0.650-0.674] versus 0.640 [0.628-0.651]).

However, the EM continued to outperform the Charlson co-morbidities in predicting 30-day mortality, even when diagnostic categories were used as individual covariables (cases: EM 0.695 [0.677-0.714] versus Charlson co-morbidities 0.632 [0.613-0.652] and controls: 0.695 [0.677-0.714) versus 0.632 [0.613-0.652)). The EM also outperformed individual Charlson co-morbidities in predicting 365-day mortality (cases: EM 0.672 [0.661$0.683]$ versus $\mathrm{CM} 0.638$ [0.627-0.649] and controls: 0.696 [0.685-0.707] versus 0.662 [0.650-0.674]). 


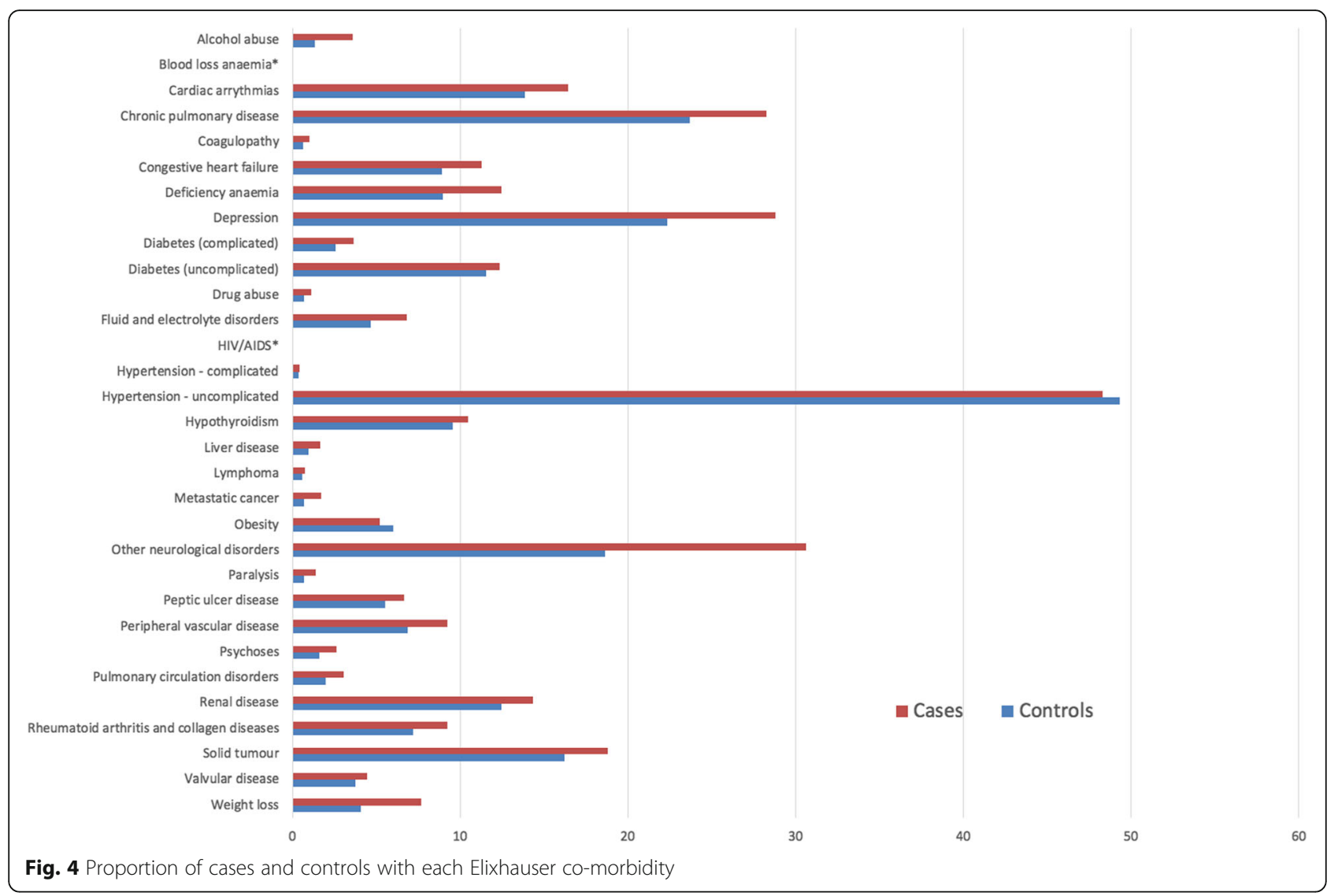

\section{Discussion}

The principal aim of this study was to transparently and reproducibly create comorbidity lists for future researchers working with Read-coded databases. The final lists are available as Additional files 1 and 2 that can be readily imported into commonly used statistical software packages (Additional file 1 and Additional file 2). These lists are particularly important for researchers analyzing UK primary care datasets such as Clinical Practice Research Datalink (CPRD) GOLD [15] and The Health Improvement Network (THIN) [16]. Although such researchers may need to include a composite comorbidity

Table 1 Diagnosis count using Charlson and Elixhauser comorbidity lists

\begin{tabular}{llllll}
\hline \multirow{2}{*}{ Count } & \multicolumn{2}{l}{ Charlson (\%) } & & \multicolumn{2}{l}{ Elixhauser (\%) } \\
\cline { 2 - 3 } & Cases & Controls & & Cases & Controls \\
\hline 0 & 27.6 & 36.6 & & 9.2 & 15.3 \\
1 & 23.8 & 23.8 & & 16.9 & 21.2 \\
2 & 18.3 & 16.9 & 19.1 & 20.8 \\
3 & 12.4 & 10.7 & 18.4 & 16.5 \\
4 & 8.0 & 5.9 & & 14.1 & 11.5 \\
5 & 4.3 & 3.1 & 9.7 & 7.2 \\
$>6$ & 5.6 & 3.0 & & 12.5 & 7.5 \\
\hline
\end{tabular}

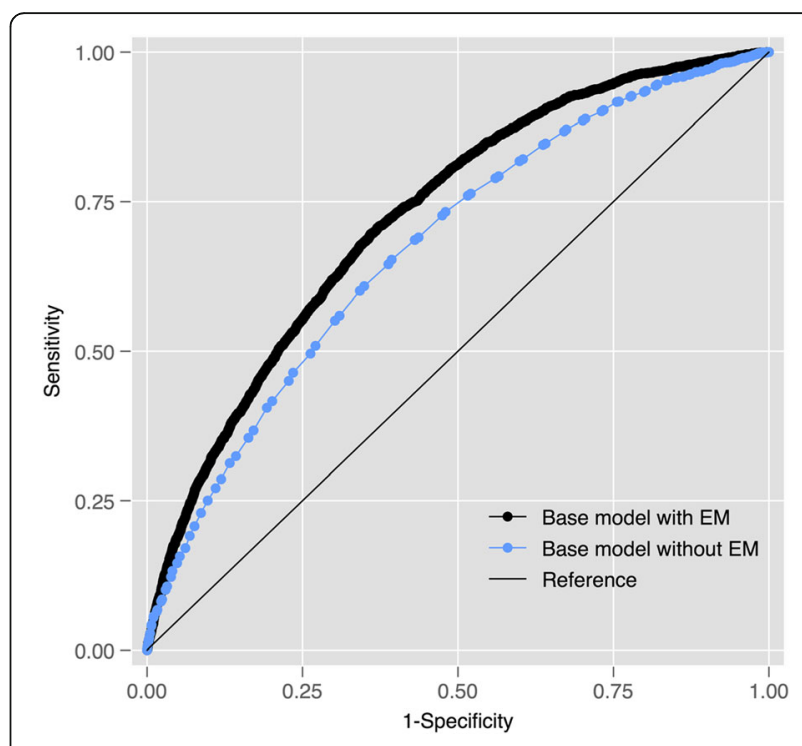

Fig. 5 Receiver-Operating Characteristic (ROC) curves for a regression model (co-variables: age and sex) predicting 365-day mortality amongst cases with and without the EM 


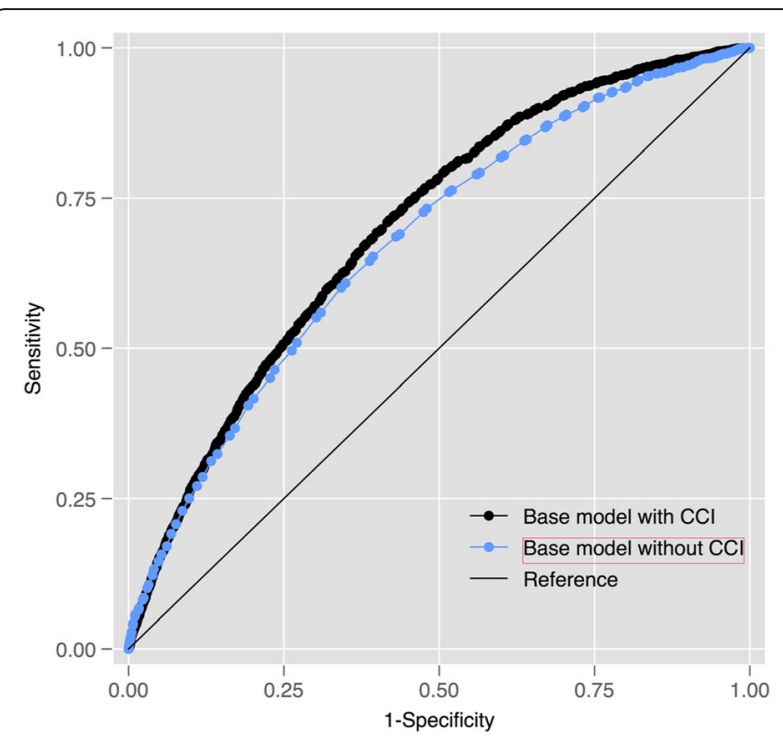

Fig. 6 Receiver-Operating Characteristic (ROC) curves for a regression model (co-variables: age and sex) predicting 365-day mortality amongst cases with and without the $\mathrm{CCl}$

score for the purposes of risk adjusting outcomes, it is onerous and time-consuming to create comprehensive code lists for indices such as the EM, which encompasses 31 individual disease categories. This is particularly difficult for Read-coded databases as the Read syntax includes codes along multiple axes, e.g. diseases, procedures, examination findings, and administrative events such as clinic referrals. Read terms also include spelling errors (e.g. "[V]Folow-up exam aft other treatment for malignant neoplasm", inconsistent abbreviations ("[X]Vit B12/folic/oth ant-megalobl-anaem caus adv ef ther use", obscure diseases (e.g. "Sequoiosis (red-cedar asthma)"), and synonyms ("Plummer - Vinson syndrome" versus "Plummer-Vinson syndrome") that can lead to codes being missed. Publiclyaccessible lists of diagnostic codes for both the CCI and EM will save analyst time and improve the reproducibility of primary care research.

We have been unable to identify any previous attempts to translate the EM (initially published using ICD-9-CM codes) for use in Read-coded databases. Although the $\mathrm{CCI}$ is the most commonly used comorbidity index in studies with administrative data [4], it has less predictive value than the EM in many populations. An earlier study [13] reported such a list for CCI but identified 3156 codes, which is only $54 \%$ of those identified by our study. Our study should not be interpreted as criticism of these authors but as an extension of their work as we used their findings - together with those published by other single disease studies - to help create our own CCI list. However, the differences between the two studies highlights the difficulties that research groups face when trying to create comprehensive lists of Read codes and employ them in adequately risk-adjusted research.

This study also showed that the EM performed better than the CCI at predicting hip fracture mortality. However, even the EM only added a modest degree of additional predictive value over and above a simple regression model with age and sex covariables. Although diagnostic codes from the entire lifetime record of patients added predictive value beyond those recorded within the preceding 1- and 5-years, this increase was modest. It is likely that comorbidities recorded within the previous 12-months will be sufficient for risk adjustment in most studies. These findings should reassure researchers that are necessarily working with limited extracts of primary care data.

\section{Limitations}

The main limitation of our study is that it is difficult to be certain that all diagnoses were included within each category. However, we did use a number of strategies to maximize our capture of relevant codes, including screening by independent clinicians and checks using lists created by other researchers for specific disease populations. It is also possible that discrepancies could arise in terms of disease classification. For example, lymphoepithelial carcinoma was categorized as "solid tumour" for the purposes of the EM rather than "lymphoma". These decisions were aided by recourse to the ICD-9-CM codes used in previous publications as well as textbooks and subject experts. It is, however, possible that some classifications will be contentious or change over time. Although this resource is likely to be sufficient for the purposes of comorbidity risk adjustment, researchers working on specific disease processes should satisfy themselves that these lists are sufficient for their purposes.

\section{Conclusion}

We have adopted a robust and transparent approach to identifying Read codes that can be used by future researchers to calculate CCI and EM. This study also showed that, although the EM outperforms the CCI and models are improved by using comorbidity codes captured over a long period of time, the differences are modest. Researchers with access to limited datasets concerning comorbidities may create logistic regression models with similar discrimination to those with access to complete healthcare records.

\section{Additional files}

Additional file 1: Read codes for Charlson co-morbidities. (CSV 417 kb) Additional file 2: Read codes for Elixhauser co-morbidities. (CSV 513 kb) Additional file 3: Screening Principles. (DOCX 120 kb) 
Additional file 4: Read codes used to identify the hip fracture cohort (DOCX $126 \mathrm{~kb}$ )

Additional file 5: Effect of using diagnostic records over different durations. (DOCX $20 \mathrm{~kb}$ )

\section{Abbreviations}

AUROC: Area under the receiver operating characteristic; CCl: Charlson Comorbidity Index; Cl: Confidence interval; CPRD: Clinical Practice Research Datalink; EM: Elixhauser Method; GafREC: Governance arrangements for Research Ethics Committees; GDPR: General Data Protection Regulation; GP: General practice; ICD-9-CM: International classification of disease, 9th edition, clinical modification; IQR: Interquartile range; ISAC: Independent Scientific Advisory Committee; MHRA: Medicines and Healthcare products Regulatory Agency; NHS: National Health Service; ROC: Receiver Operating Characteristic; THIN: The Health Improvement Network; UK: United Kingdom; UTS: Up to standard

\section{Acknowledgements}

We are grateful to the authors (cited in the text) whose comorbidity code lists contributed to this resource and to GP practices participating in the CPRD for providing data.

\section{Authors' contributions}

DM designed the study, created the code lists, undertook the data analysis, and drafted the manuscript. JM created the code lists contributed to the study design, interpretation of the data, and made critical revisions to the manuscript. AD prepared a dataset from individual CPRD data files, helped check the code lists, contributed to interpretation of the data, and made critical revisions to the manuscript. AJ, DP, CZ, BG, and MC contributed to the study design, interpretation of the data, and critically revised the manuscript. All authors have read and approved the final manuscript.

\section{Authors' information}

DM and JM are a Clinical Research Fellows in Musculoskeletal Trauma at the University of Oxford, AD is a computer scientist and Senior Database Manager at the University of Oxford, AJ is Professor of Translational Statistics at the University of Bristol, DP is Associate Professor of Orthopaedics \& Trauma Surgery at the University of Oxford, CZ is a MD-PhD candidate and Health Law Fellow at Yale University (Connecticut, USA), BG is Head of the Pre-Hospital, Emergency and Trauma Research Unit at Monash University (Melbourne, Australia), and MC is Professor of Orthopaedic Trauma Surgery at the University of Oxford.

\section{Funding}

David Metcalfe is funded by an Oxford-UCB Fellowship in Biomedical Research. No funding body had any influence over the design or conduct of the research, or the decision to publish.

\section{Availability of data and materials}

The data that support the findings of this study are available from the Clinical Practice Research Datalink (CPRD) but restrictions apply to the availability of these data, which were used under license for the current study, and so are not publicly available. Data may however be available on application directly to the CPRD.

\section{Ethics approval and consent to participate}

Ethical approval was not sought in line with the latest Governance Arrangements for Research Ethics Committees (GafREC) guidance. Approval to use the data was provided by the Independent Scientific Advisory Committee (ISAC) at the MHRA (ISAC Protocol No. 13_069RA). Personal data was processed under Articles $6(1)(f)$ and $9(1)(f)$ of the General Data Protection Regulation (EU 2016/6709).

\section{Consent for publication}

Not applicable.

\section{Competing interests}

None.

\section{Author details}

Oxford Trauma, Kadoorie Centre for Critical Care Research and Education, Nuffield Department of Orthopaedics, Rheumatology and Musculoskeletal Sciences (NDORMS), John Radcliffe Hospital, Headley Way, Oxford OX3 9BU, UK. ${ }^{2}$ Centre for Statistics in Medicine, NDORMS, Nuffield Orthopaedic Centre, University of Oxford, Windmill Road, Oxford OX3 7LD, UK. ${ }^{3}$ Musculoskeletal Research Unit, Translational Health Sciences, Bristol Medical School, University of Bristol, Learning and Research Building, Level 1, Southmead Hospital, Bristol BS10 5NB, UK. ${ }^{4}$ National Institute for Health Research Bristol Biomedical Research Centre (NIHR Bristol BRC), University Hospitals Bristol NHS Foundation Trust, University of Bristol, Southmead Hospital, Bristol BS10 5NB, UK. ${ }^{5} \mathrm{MRC}$ Lifecourse Epidemiology Unit, University of Southampton, Southampton General Hospital, Southampton SO16 6YD, UK. ${ }^{6}$ Yale School of

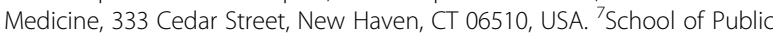
Health and Preventive Medicine, Monash University, Level 3, 553 St Kilda Road, Melbourne, VIC 3004, Australia.

Received: 22 August 2018 Accepted: 20 May 2019

Published online: 06 June 2019

\section{References}

1. Charlson ME, Pompei P, Ales KL, et al. A new method of classifying prognostic comorbidity in longitudinal studies: development and validation. J Chronic Dis. 1987:40(5):373-83.

2. Elixhauser A, Steiner C, Harris DR, et al. Comorbidity measures for use with administrative data. Med Care. 1998;36(1):8-27.

3. Molto A, Dougados M. Comorbidity indices. Clin Exp Rheumatol. 2014;32(5 Suppl 85):S-131-4

4. Sharabiani MT, Aylin P, Bottle A. Systematic review of comorbidity indices for administrative data. Med Care. 2012;50(12):1109-18. https://doi.org/10. 1097/MLR.0b013e31825f64d0.

5. Karres J, Heesakkers NA, Ultee JM, et al. Predicting 30-day mortality following hip fracture surgery: evaluation of six risk prediction models. Injury. 2015;46(2):371-7. https://doi.org/10.1016/j.injury.2014.11.004.

6. Quan H, Li B, Couris CM, et al. Updating and validating the Charlson comorbidity index and score for risk adjustment in hospital discharge abstracts using data from 6 countries. Am J Epidemiol. 2011;173(6):676-82. https://doi.org/10.1093/aje/kwq433.

7. Menendez ME, Ring D, Harris MB, et al. Predicting in-hospital mortality in elderly patients with cervical spine fractures: a comparison of the Charlson and Elixhauser comorbidity measures. Spine (Phila Pa 1976). 2015;40(11): 809-15. https://doi.org/10.1097/BRS.0000000000000892.

8. Menendez ME, Ring D. A comparison of the Charlson and Elixhauser comorbidity measures to predict inpatient mortality after proximal Humerus fracture. J Orthop Trauma. 2015;29(11):488-93. https://doi.org/10.1097/BOT. 0000000000000380

9. Quan H, Sundararajan V, Halfon $\mathrm{P}$, et al. Coding algorithms for defining comorbidities in ICD-9-CM and ICD-10 administrative data. Med Care. 2005: 43(11):1130-9.

10. D'Hoore W, Bouckaert A, Tilquin C. Practical considerations on the use of the Charlson comorbidity index with administrative data bases. J Clin Epidemiol. 1996:49(12):1429-33.

11. Deyo RA, Cherkin DC, Ciol MA. Adapting a clinical comorbidity index for use with ICD-9-CM administrative databases. J Clin Epidemiol. 1992; 45(6):613-9.

12. Romano PS, Roos $L L$, Jollis JG. Adapting a clinical comorbidity index for use with ICD-9-CM administrative data: differing perspectives. J Clin Epidemiol. 1993;46(10):1075-9 discussion 81-90.

13. Khan NF, Perera R, Harper $\mathrm{S}$, et al. Adaptation and validation of the Charlson index for read/OXMIS coded databases. BMC Fam Pract. 2010;11:1. https:// doi.org/10.1186/1471-2296-11-1.

14. Benson $\mathrm{T}$. The history of the read codes: the inaugural James read memorial lecture 2011. Inform Prim Care. 2011:19(3):173-82.

15. Herrett E, Gallagher AM, Bhaskaran K, et al. Data resource profile: clinical practice research datalink (CPRD). Int J Epidemiol. 2015;44(3):827-36. https:// doi.org/10.1093/ije/dyv098.

16. Denburg MR, Haynes K, Shults J, et al. Validation of the health improvement network (THIN) database for epidemiologic studies of chronic kidney disease. Pharmacoepidemiol Drug Saf. 2011;20(11):1138-49. https://doi.org/10.1002/ pds.2203. 
17. U.S. National Center for Health Statistics. International Statistical Classification of Diseases, Clinical Modification (ICD-9-CM). Hyattsville: National Center for Health Statistics; 2008.

18. Clinical Practice Research Datalink (CPRD). Medical Dictionary. London: Department of Health and Social Care; 2018.

19. ClinicalCodes.org. Manchester: University of Manchester; 2017. https:// clinicalcodes.rss.mhs.man.ac.uk. Accessed 1 Oct 2018.

20. Carr MJ, Ashcroft DM, Kontopantelis E, et al. Premature death among primary care patients with a history of self-harm. Ann Fam Med. 2017;15(3): 246-54. https://doi.org/10.1370/afm.2054.

21. Doyle $M$, While $D$, Mok $P L$, et al. Suicide risk in primary care patients diagnosed with a personality disorder: a nested case control study. BMC Fam Pract. 2016;17:106. https://doi.org/10.1186/s12875-016-0479-y.

22. Fairhurst C, Watt I, Martin F, et al. Exposure to sodium channel-inhibiting drugs and cancer survival: protocol for a cohort study using the QResearch primary care database. BMJ Open. 2014;4(11):e006604. https://doi.org/10. 1136/bmjopen-2014-006604.

23. Kontopantelis E, Springate $D$, Reeves $D$, et al. Withdrawing performance indicators: retrospective analysis of general practice performance under UK quality and outcomes framework. BMJ. 2014;348:g330. https://doi.org/10. 1136/bmj.g330.

24. Muller S, Hider SL, Raza K, et al. An algorithm to identify rheumatoid arthritis in primary care: a clinical practice research datalink study. BMJ Open. 2015; 5(12):e009309. https://doi.org/10.1136/bmjopen-2015-009309.

25. Nicholson A, Ford E, Davies KA, et al. Optimising use of electronic health records to describe the presentation of rheumatoid arthritis in primary care: a strategy for developing code lists. PLoS One. 2013;8(2):e54878. https://doi. org/10.1371/journal.pone.0054878.

26. Reeves D, Springate DA, Ashcroft DM, et al. Can analyses of electronic patient records be independently and externally validated? The effect of statins on the mortality of patients with ischaemic heart disease: a cohort study with nested case-control analysis. BMJ Open. 2014;4(4):e004952. https://doi.org/10.1136/bmjopen-2014-004952.

27. Reilly S, Olier I, Planner C, et al. Inequalities in physical comorbidity: a longitudinal comparative cohort study of people with severe mental illness in the UK. BMJ Open. 2015;5(12):e009010. https://doi.org/10.1136/bmjopen2015-009010.

28. Stocks SJ, Kontopantelis E, Akbarov A, et al. Examining variations in prescribing safety in UK general practice: cross sectional study using the clinical practice research datalink. BMJ. 2015;351:h5501. https://doi.org/10. 1136/bmj.h5501.

29. Thompson A, Ashcroft DM, Owens L, et al. Drug therapy for alcohol dependence in primary care in the UK: a clinical practice research datalink study. PLoS One. 2017;12(3):e0173272. https://doi.org/10.1371/journal.pone. 0173272.

30. Zhong WW, Juhaeri J, Cole SR, et al. HbA1C variability and hypoglycemia hospitalization in adults with type 1 and type 2 diabetes: a nested casecontrol study. J Diabetes Complicat. 2018;32(2):203-9. https://doi.org/10. 1016/j.jdiacomp.2017.10.008.

31. Cleves MA. From the help desk: comparing areas under receiver operating characteristic curves from two or more probit or logit models. Stata J. 2002; 2(3):301-13.

32. NHS Health Research Authority (HRA). Governance arrangements for research ethics committees. London: NHS Health Research Authority; 2018. https://www.hra.nhs.uk/documents/1560/GAfREC_2018_v1.0_FINAL_ 20180615.pdf

\section{Publisher's Note}

Springer Nature remains neutral with regard to jurisdictional claims in published maps and institutional affiliations.

Ready to submit your research? Choose BMC and benefit from:

- fast, convenient online submission

- thorough peer review by experienced researchers in your field

- rapid publication on acceptance

- support for research data, including large and complex data types

- gold Open Access which fosters wider collaboration and increased citations

- maximum visibility for your research: over $100 \mathrm{M}$ website views per year

At $\mathrm{BMC}$, research is always in progress.

Learn more biomedcentral.com/submissions 